\title{
THE
}

\section{Skyrmion physics beyond the lowest Landau-level approximation}

V. Melik-Alaverdian

University of Rhode Island

N. E. Bonesteel

G. Ortiz

Follow this and additional works at: https://digitalcommons.uri.edu/phys_facpubs

Terms of Use

All rights reserved under copyright.

\section{Citation/Publisher Attribution}

Melik-Alaverdian, V., Bonesteel, N. E., \& Ortiz, G. (1999). Skyrmion physics beyond the lowest Landau-level approximation. Phys. Rev. B, 60, R8501--R8504. doi: 10.1103/PhysRevB.60.R8501

Available at: https://doi.org/10.1103/PhysRevB.60.R8501

This Article is brought to you for free and open access by the Physics at DigitalCommons@URI. It has been accepted for inclusion in Physics Faculty Publications by an authorized administrator of DigitalCommons@URI. For more information, please contact digitalcommons-group@uri.edu. 


\title{
Skyrmion physics beyond the lowest Landau-level approximation
}

\author{
V. Melik-Alaverdian \\ Department of Physics, University of Rhode Island, Kingston, Rhode Island 02881 \\ N. E. Bonesteel \\ National High Magnetic Field Laboratory and Department of Physics, Florida State University, Tallahassee, Florida 32310 \\ G. Ortiz \\ Theoretical Division, Los Alamos National Laboratory, P.O. Box 1663, Los Alamos, New Mexico 87545
}

(Received 16 June 1999)

\begin{abstract}
The effects of Landau-level mixing and finite thickness of the two-dimensional electron gas on the relative stability of Skyrmion and single spin-flip excitations at Landau-level filling factor $\nu=1$ have been investigated. Landau-level mixing is studied by fixed-phase diffusion Monte Carlo, and finite thickness is included by modifying the effective Coulomb interaction. Both Landau-level mixing and finite thickness lower Skyrmion excitation energies and favor Skyrmions with fewer spin flips. However, the two effects do not work "coherently." When finite thickness is included, the effect of Landau-level mixing is strongly suppressed. [S0163-1829(99)52136-X]
\end{abstract}

In a seminal paper, Sondhi et al. ${ }^{1}$ predicted that if the ratio of the Zeeman energy to the exchange energy is sufficiently small, the quasiparticle excitations of the $\nu=1$ integer quantum Hall state should be charged "Skyrmions" with topologically nontrivial spin textures. ${ }^{1}$ The existence of these exotic excitations is now well confirmed by experiment. ${ }^{2-4}$

While the initial theoretical description of Skyrmions in terms of an effective long-wavelength nonlinear $\sigma$ model is appropriate for excitations whose magnetization varies slowly on the scale of the magnetic length; in typical experiments the number of reversed spins per Skyrmion is $\sim 3-4$ and a more microscopic description is required. A step towards such a description was taken by MacDonald, Fertig, and Brey, ${ }^{5}$ who introduced explicit wave functions describing Skyrmions with well-defined spin quantum numbers.

In this paper we present the results of calculations in which these microscopic wave functions have been used to study the effects of finite thickness of the two-dimensional electron gas (2DEG) and Landau-level mixing on $\nu=1$ Skyrmion states. Finite thickness is included by modifying the Coulomb interaction and Landau-level mixing is studied using the fixed-phase diffusion Monte Carlo method. ${ }^{6,7}$ One motivation for this work is the observation by Schmeller et $a l .{ }^{4}$ of Skyrmion energy gaps measured in transport experiments which are approximately a factor of 4 smaller than theoretical estimates based on lowest Landau-level calculations, ${ }^{8}$ a result which led these authors to speculate that Landau-level mixing plays an important role in reducing Skyrmion excitation energies. In our calculations, we find that both finite thickness and Landau-level mixing do in fact lower Skyrmion excitation energies and favor Skyrmions with fewer spin flips. However, the two effects do not work efficiently together-the finite thickness effect strongly suppresses the Landau-level mixing effect.

In our calculations, we have used the spherical geometry ${ }^{9}$ in which $N$ electrons with charge $-e$ and mass $m$ are confined to move on the surface of a sphere of radius $R$ in the presence of a radial magnetic field $B$. The total number of flux quanta piercing the surface of the sphere is then $N_{\phi}$ $=2\left(R / l_{0}\right)^{2}$, where $l_{0}=\sqrt{\hbar c / e B}$ is the magnetic length, and the Hamiltonian describing the system is

$$
H=\frac{1}{2 m} \sum_{i=1}^{N}\left(\hat{\mathbf{r}}_{i} \times\left(\frac{\hbar}{i} \nabla_{i}+\frac{e}{c} \mathbf{A}_{i}\right)\right)^{2}+V_{C}+H_{Z} .
$$

Here, $V_{C}=\Sigma_{i<j} V\left(r_{i j}\right)$ is the Coulomb interaction where $r_{i j}$ is the chord distance between electrons $i$ and $j$, and $H_{Z}$ $=-g \mu_{B} \sum_{i} S_{i z} B$ is the Zeeman interaction. We work in the Wu-Yang gauge in which the vector potential is $\mathbf{A}$ $=\mathbf{e}_{\phi} \hbar c N_{\phi}(1-\cos \theta) /(2 e R \sin \theta) .{ }^{10}$ The softening of the short-range part of the Coulomb interaction due to finite thickness of the lowest subband wave function of the 2DEG is included through the modified interaction $V(r)$ $=e^{2} / \epsilon \sqrt{r^{2}+\beta^{2}}$, where $\beta$ is a parameter characterizing the thickness. ${ }^{11}$

For the $\nu=1$ ground state, the electron spins are fully polarized and the lowest Landau level is completely full. On the sphere, this occurs when $N=N_{\phi}+1$ and the corresponding wave function is a Slater determinant of lowest Landaulevel wave functions. If the electron positions are given in terms of the complex coordinate $z=\tan (\theta / 2) \exp (-i \phi)$, where $\theta$ and $\phi$ are the usual spherical angles, then, exploiting the Vandermonde form of the Slater determinant, the ground-state wave function can be written

$$
\psi_{g s}=U^{N} \phi^{\prime 2} \prod_{i<j}\left(z_{i}-z_{j}\right) \otimes\left(\uparrow_{1} \uparrow_{2} \cdots \uparrow_{N}\right),
$$

where $U=\Pi_{k}\left(1+\left|z_{k}\right|^{2}\right)^{-1}$

Single spin-flip excitations of the system are constructed by removing a spin-up electron from the ground state, flipping its spin, and reintroducing it into the lowest spin-down Landau level. Letting $K$ denote the number of reversed spins associated with a given quasiparticle excitation, this proce- 
dure produces a $K=0$ quasihole with charge $+e$ and a $K$ $=1$ quasielectron with charge $-e$. The transport gap which determines the activated temperature dependence of the longitudinal resistivity at $\nu=1$ is set by the excitation energy for creating a quasielectron and quasihole with infinite separation. On the sphere, the best approximation to this state can be realized by placing the quasihole at the bottom of the sphere $(\theta=\pi)$ and the quasielectron at the top of the sphere $(\theta=0)$. The corresponding wave function is

$$
\psi_{s f}=\mathcal{A} U^{N} \phi^{\prime 2} \prod_{i<j, i \neq 1}\left(z_{i}-z_{j}\right) \otimes\left(\downarrow_{1} \uparrow_{2} \cdots \uparrow_{N}\right),
$$

where the operator $\mathcal{A}$ antisymmetrizes the wave function under the exchange of all pairs of electrons.

The nature of the quasiparticle excitations at $\nu=1$ depends on the relative size of the Zeeman energy and the exchange energy characterized by the dimensionless ratio $\widetilde{g}$ $=g \mu_{B} B /\left(e^{2} / \epsilon l_{0}\right)$. In the limit of large $\tilde{g}$, the transport gap for the $\nu=1$ integer quantum Hall state is set by the single spin-flip excitation described above. However, as $\widetilde{g}$ is lowered below a critical value, the single spin flip becomes unstable to the formation of a neutral Skyrmion pair, i.e., a well-separated charge $+e$ and charge $-e$ Skyrmion pair, involving more than one flipped spin. ${ }^{1}$ A microscopic wave function description of the charge $+e$ Skyrmion excitations was developed by MacDonald, Fertig, and Brey, ${ }^{5}$ who showed that for a model Hamiltonian with a hard-core $\delta$ function repulsion, the states

$$
\begin{aligned}
\psi_{s k}(K)= & \mathcal{A} U^{\left(N_{\phi}+1\right) / 2} \prod_{i<j}\left(z_{i}-z_{j}\right) z_{K+1} z_{K+2} \cdots z_{N} \\
& \otimes\left(\downarrow_{1} \cdots \downarrow_{K} \uparrow_{K+1} \cdots \uparrow_{N}\right)
\end{aligned}
$$

are exact eigenstates of $H$ with charge $e$ and $K$ reversed spins.

The wave functions $\psi_{g s}, \psi_{s f}$, and $\psi_{s k}(K)$ describe states in which the kinetic energy is completely quenched and there is no Landau-level mixing. Therefore, the only contributions to the energy gaps computed using these wave functions will come from the Coulomb and Zeeman energies. The variational result for the Coulomb contribution to the single spinflip energy gap is

$$
\Delta_{s f}=\left\langle\psi_{s f}\left|V_{C}\right| \psi_{s f}\right\rangle-\left\langle\psi_{g s}\left|V_{C}\right| \psi_{g s}\right\rangle .
$$

In the absence of Landau-level mixing, an exact particle-hole symmetry in the lowest Landau level transforms a charge $+e$ Skyrmion with $K$ reversed spins into a charge $-e$ Skyrmion with $K+1$ reversed spins. It is therefore possible to compute the energy gap for creating a neutral Skyrmion pair by first computing $\Delta_{s f}$ and then computing the change in Coulomb energy of the charge $+e$ Skyrmion as the number of reversed spins is increased,

$$
\delta(K)=\left\langle\psi_{s k}(K)\left|V_{C}\right| \psi_{s k}(K)\right\rangle-\left\langle\psi_{s k}(0)\left|V_{C}\right| \psi_{s k}(0)\right\rangle .
$$

As pointed out by Abolfath et al., ${ }^{12}$ the wave functions (4) involve power-law tails which lead to strong finite-size effects. In our variational calculations, we have computed $\delta(K)$ for $\beta=0$ and $\beta=l_{0}$ by doing a careful extrapolation to

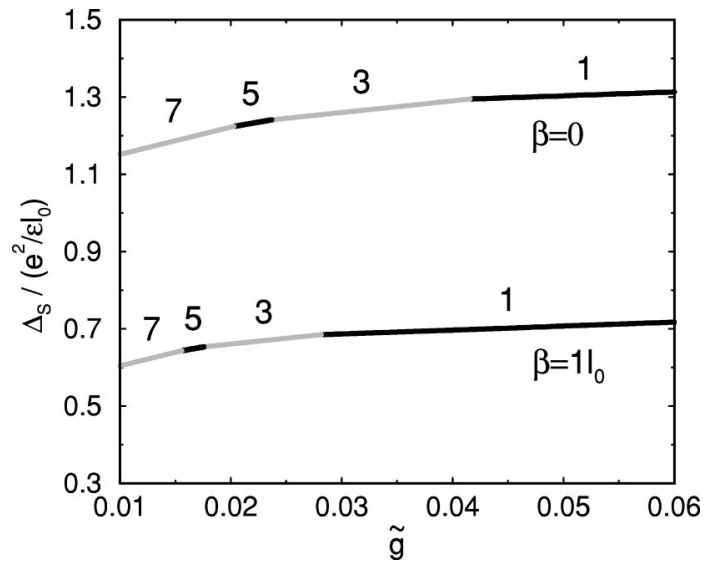

FIG. 1. Energy gaps for creating neutral Skyrmion pairs plotted vs $\widetilde{g}$. The number labeling each line segment is the total number of reversed spins $S=2 K+1$. Results are given for thickness parameter $\beta=0$ and $\beta=l_{0}$ and have been extrapolated to the thermodynamic limit.

the thermodynamic limit, considering systems with up to 100 electrons. For $\beta=0$, our results agree with previous calculations of $\delta(K)$. $^{12,13}$

When the Zeeman contribution is included, the total energy gap for creating a neutral Skyrmion pair for which the total number of reversed spins $S=2 K+1$ is

$$
\Delta_{S}(\widetilde{g})=\Delta_{s f}+2 \delta(K)+S \widetilde{g} \frac{e^{2}}{\epsilon l_{0}} .
$$

These gaps are plotted vs. $\widetilde{g}$ in Fig. 1 for $S=1,3,5$, and 7 and for $\beta=0$ and $\beta=l_{0}$. In contrast to Hartree-Fock calculations ${ }^{8}$ which describe continuously varying spin textures, our results show the quantum nature of the Skyrmion. As $\widetilde{g}$ decreases, the energy gap undergoes level crossings in which the total number of reversed spins of the neutral Skyrmion pair jumps by two. Note that although we have extended the $S=7$ line down to $\widetilde{g}=0.01$, we expect that as $\widetilde{g}$ decreases, the density of level crossings will increase until the energy gap becomes, effectively, a smooth function of $\widetilde{g}$.

Increasing the thickness of the 2DEG is seen to lower the Skyrmion energy gaps and favors excitations with fewer spin flips. This effect can be understood qualitatively by noting that as the number of reversed spins $K$ increases, the charge of the Skyrmion spreads out, resulting in a lowering of its Coulomb energy. When the thickness is increased, and consequently the short-range part of the electron-electron repulsion is decreased, the excitation energies of the larger Skyrmions will decrease less than those of the smaller Skyrmions.

We now turn to the effect of Landau-level mixing on the Skyrmion energy gaps. The importance of Landau-level mixing in a given system is characterized by the electron gas parameter $r_{s}=1 /\left(a_{B} \sqrt{\pi n}\right)$, where $n$ is the carrier density and $a_{B}=\epsilon \hbar^{2} / m e^{2}$ is the effective Bohr radius. It is straightforward to show that $r_{s}=(\nu / 2)^{1 / 2}\left(e^{2} / \epsilon l_{0}\right) / \hbar \omega_{c}$, where $\hbar \omega_{c}$ $=\hbar e B / m c$ is the cyclotron energy so that, at fixed $\nu$, in the limit $r_{s} \rightarrow 0$, the cyclotron energy is much larger than the Coulomb energy and there is no Landau-level mixing. However, in typical experiments, $r_{s}$ is of order 1 or higher and Landau-level mixing cannot be ignored. 


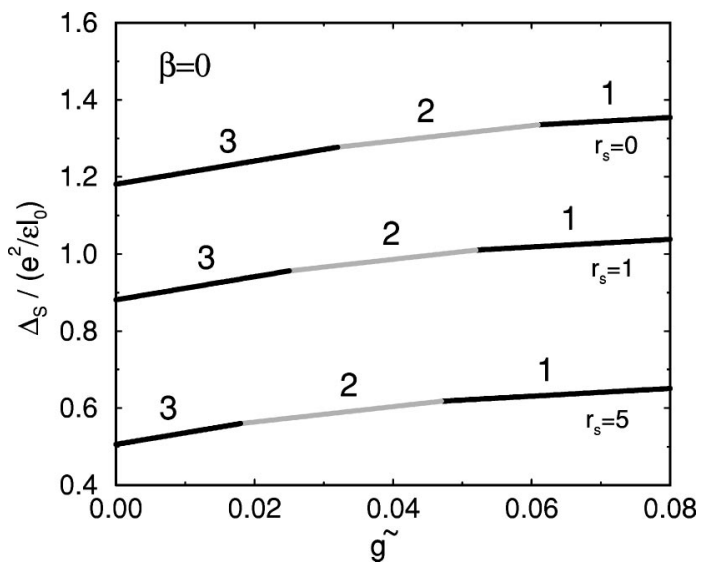

FIG. 2. Energy gaps for creating neutral excitations consisting of a charge $-e$ single spin-flip quasielectron and a charge $+e$ Skyrmion with $K=0,1$, and 2 reversed spins for $r_{s}=0,1$, and 5 as a function of $\widetilde{g}$ for thickness parameter $\beta=0$. Each line segment is labeled by the total number of reversed spins $S=K+1$. Results are for a 30 electron system.

To study Landau-level mixing, we have used the fixedphase diffusion Monte Carlo method, ${ }^{6}$ which has proven to be a useful tool for studying the effect of Landau-level mixing on quantum Hall states. ${ }^{6,7}$ All of the wave functions considered here have been given in the form

$$
\psi=\mathcal{A} \psi_{\text {space }}\left(\mathbf{r}_{1}, \cdots, \mathbf{r}_{N}\right) \otimes\left(\downarrow_{1} \cdots \downarrow_{K} \uparrow_{K+1} \cdots \uparrow_{N}\right),
$$

where $\psi_{\text {space }}$ is completely antisymmetric under the exchange of any pair of electrons $i$ and $j$, where either $1 \leqslant i, j \leqslant K$ or $K+1 \leqslant i, j \leqslant N$. In a fixed-phase diffusion Monte Carlo simulation, these wave functions are used as trial states by first writing the space part of the wave function in the form

$$
\psi_{\text {space }}\left(\mathbf{r}_{1}, \cdots, \mathbf{r}_{N}\right)=\left|\psi_{\text {space }}\left(\mathbf{r}_{1}, \cdots, \mathbf{r}_{N}\right)\right| e^{i \varphi\left(\mathbf{r}_{1}, \cdots, \mathbf{r}_{N}\right)},
$$

then "fixing" the trial phase $\varphi$, and finally constructing an effective bosonic Schrödinger equation for the positive definite wave function $\left|\psi_{\text {space }}\right|$ and solving that equation using standard diffusion Monte Carlo techniques. ${ }^{13,14}$ The result of this procedure is the lowest energy state of the form (8) subject to the constraint that the phase is the same as that of the trial function.

We have implemented this procedure using $\psi_{g s}, \psi_{s f}$, and $\psi_{s k}(K)$ as trial wave functions to obtain, respectively, the fixed-phase energies $E_{g s}^{F P}, E_{s f}^{F P}$, and $E_{s k}^{F P}(K)$ for a system with 30 electrons. For finite $r_{s}$, it is no longer possible to transform a charge $+e$ Skyrmion into a charge $-e$ Skyrmion by particle-hole symmetry. Therefore, in order to calculate a physical quantity, we have computed the energy gaps for creating a charge $+e$ Skyrmion with $K$ spin flips and a single spin-flip quasielectron. As before, letting $\Delta_{s f}$ $=E_{s f}^{F P}-E_{g s}^{F P}$ and $\delta(K)=E_{s k}^{F P}(K)-E_{s k}^{F P}(0)$, the corresponding energy gaps are given by

$$
\Delta_{S}(\widetilde{g})=\Delta_{s f}+\delta(K)+S \widetilde{g} \frac{e^{2}}{\epsilon l_{0}},
$$

where now $S=K+1$.

These energy gaps are plotted as a function of $\widetilde{g}$ in Fig. 2 for $S=1,2$, and 3 and for $r_{s}=0,1$, and 5. As $r_{s}$ is increased,

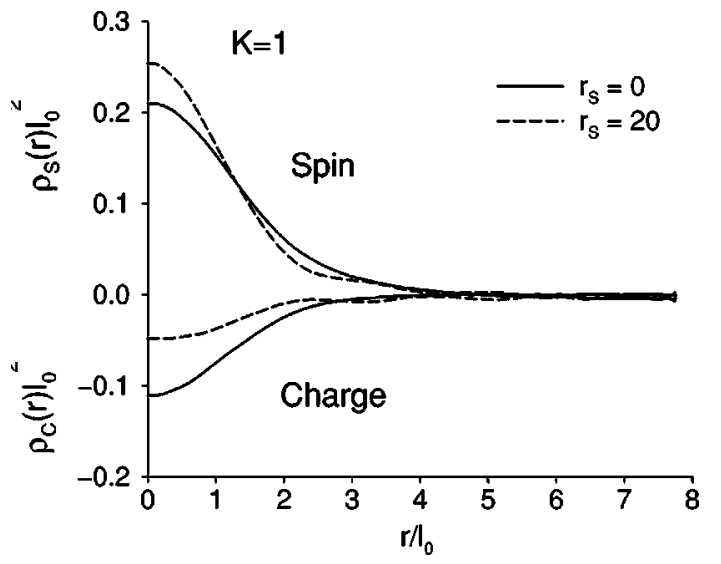

FIG. 3. Spin and charge densities as a function of $r / l_{0}(r$ is the chord distance on the sphere from the center of the Skyrmion) for a charge $+e$ Skyrmion with $K=1$ for $r_{s}=0$ and $r_{s}=20$. Results are for a 30 electron system.

the effect of Landau-level mixing is seen to be qualitatively similar to that of finite thickness-the Skyrmion excitation energies are lowered, with the smaller size Skyrmions having their energies lowered the most. This effect can be understood qualitatively by examining the effect of Landau-level mixing on the Skyrmion spin and charge densities $\rho_{s}$ and $\rho_{c}$ defined by

$$
\rho_{c}^{s}(\mathbf{r})=\frac{\left\langle\psi_{s k}(K)\left|\left(\rho_{\downarrow}(\mathbf{r}) \mp \rho_{\uparrow}(\mathbf{r})\right)\right| \psi_{s k}(K)\right\rangle}{\left\langle\psi_{s k}(K) \mid \psi_{s k}(K)\right\rangle} \pm \bar{\rho},
$$

where $\rho_{\sigma}$ is the number density operator for spin $\sigma$ and $\bar{\rho}$ is the uniform number density far from the Skyrmion. Figure 3 shows mixed estimates ${ }^{14}$ of $\rho_{s}$ and $\rho_{c}$ for the $K=1$ charge $+e$ Skyrmion for $r_{s}=0$ and $r_{s}=20$. For $r_{s}=20$, the possibility of mixing in higher Landau levels leads to a spreading out of the charge density of the Skyrmion resulting in a lowering of its Coulomb energy at the cost of some kinetic energy. This effect is suppressed as $K$ increases, because for larger values of $K$ the charge is already well spread out and there is less energy to be gained by allowing the charge to spread further.

In Fig. 4, the energy gaps are shown for $S=1,2$, and 3 and $r_{s}=0,1$, and 5 for thickness parameter $\beta=l_{0}$. When finite thickness is included, the Landau-level mixing effect is seen to be much weaker than it is for zero thickness. For example, for $\widetilde{g} \simeq 0.06$, when $\beta=0$ the $r_{s}=1$ energy gap is $\sim 25 \%$ smaller than its $r_{s}=0$ value, and when thickness is included, the $r_{s}=0$ energy gap drops $\sim 45 \%$ for $\beta=l_{0}$, but the additional reduction of the energy gap due to Landaulevel mixing is only $\sim 5 \%$ when $r_{s}=1$.

This weakening of the Landau-level mixing effect due to finite thickness can be understood as follows. The thickness correction softens the short-range part of the Coulomb interaction, which in turn reduces the interaction energy the Skyrmion stands to gain by delocalizing its charge. It follows that as $\beta$ increases, the quasielectron charge delocalizes less for a given value of $r_{s}$, and so the reduction of the energy gap decreases. Because of this suppression of the Landau-level mixing effect by finite thickness, we believe that the experimental observation by Schmeller et al. ${ }^{4}$ of Skyrmion energy gaps, which are significantly smaller than theoretical esti- 


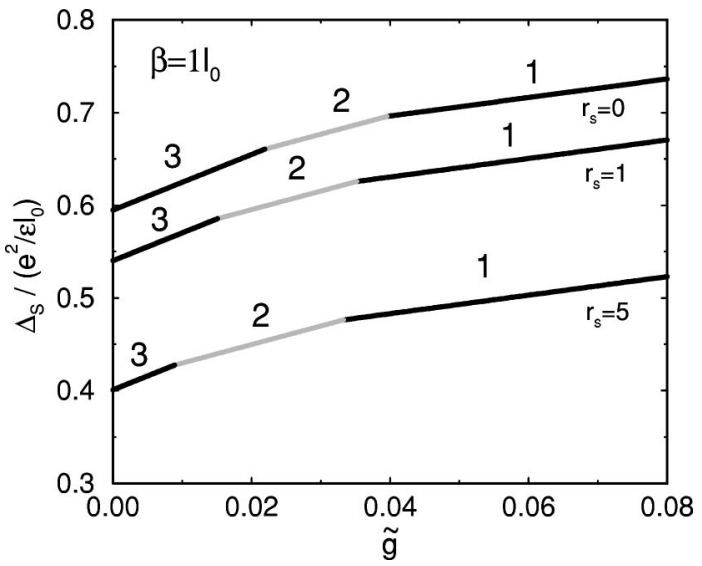

FIG. 4. Energy gaps for creating neutral excitations consisting of a charge $-e$ single spin-flip quasielectron and a charge $+e$ Skyrmion, with $K=0,1$, and 2 reversed spins for $r_{s}=0,1$, and 5 as a function of $\widetilde{g}$ for thickness parameter $\beta=l_{0}$. Each line segment is labeled by the total number of reversed spins $S=K+1$. Results are for a 30 electron system.

mates which ignore Landau-level mixing, is more likely to be due to disorder effects, which are poorly understood, than to Landau-level mixing. Note that a similar reduction of the Landau-level mixing effect due to finite thickness has been observed for spin-polarized Laughlin quasielectron and quasihole excitations in the fractional quantum Hall effect. ${ }^{7}$

It is apparent from Figs. 2 and 4 that although finite thickness strongly suppresses Landau-level mixing, the opposite is not the case. This is consistent with the observations of Králik et al. ${ }^{15}$ who performed variational Monte Carlo calculations of the effect of Landau-level mixing and finite thickness on the $\nu=1$ single spin-flip energy gap $\Delta_{s f}$, finding that for $r_{s}=1$ the inclusion of finite thickness, of roughly the same size as that considered here, significantly reduced the energy gap. Based on this observation, Králik et al. concluded that both finite thickness and Landau-level mixing contribute equally to the reduction of the energy gap. However, we believe that if Králik et al. had first included finite thickness and then Landau-level mixing, they would have observed the same strong suppression of the Landau-level mixing effect reported here.

To summarize, using Skyrmion trial states recently introduced by MacDonald, Fertig, and Brey, ${ }^{5}$ we have performed variational and fixed-phase diffusion Monte Carlo calculations of the effect of finite thickness and Landau-level mixing on Skyrmion excitations at $\nu=1$. We find that both effects lower the Skyrmion excitation energies and stabilize Skyrmions with fewer spin flips. However, we also find that the two effects do not work coherently together-when finite thickness is included, the effect of Landau-level mixing is strongly suppressed.

We thank S. P. Shukla for useful discussions. This work was supported in part by U.S. DOE Grant No. DE-FG0297ER45639 and NSF Grant No. DMR-9725080. N.E.B. acknowledges support from the Alfred P. Sloan Foundation and G.O. was supported by the U.S. DOE.
${ }^{1}$ S. L. Sondhi et al., Phys. Rev. B 47, 16419 (1993).

${ }^{2}$ S. E. Barrett et al., Phys. Rev. Lett. 74, 5112 (1995).

${ }^{3}$ E. H. Aifer, B. B. Goldberg, and D. A. Broido, Phys. Rev. Lett. 76, 680 (1996).

${ }^{4}$ A. Schmeller et al., Phys. Rev. Lett. 75, 4290 (1995).

${ }^{5}$ A. H. MacDonald, H. A. Fertig, and L. Brey, Phys. Rev. Lett. 76, 2153 (1996).

${ }^{6}$ G. Ortiz, D. M. Ceperley, and R. M. Martin, Phys. Rev. Lett. 71, 2777 (1993).

${ }^{7}$ V. Melik-Alaverdian, N. E. Bonesteel, and G. Ortiz, Phys. Rev. Lett. 79, 5286 (1997).
${ }^{8}$ H. A. Fertig et al., Phys. Rev. B 50, 11018 (1994).

${ }^{9}$ F. D. M. Haldane, Phys. Rev. Lett. 51, 605 (1983).

${ }^{10}$ T. T. Wu and C. N. Yang, Nucl. Phys. B 107, 365 (1976).

${ }^{11}$ F. C. Zhang and S. Das Sarma, Phys. Rev. B 33, 2903 (1986).

${ }^{12}$ M. Abolfath et al., Phys. Rev. B 56, 6795 (1997).

${ }^{13}$ R. K. Kamilla, X. G. Wu, and J. K. Jain, Solid State Commun. 99, 289 (1996).

${ }^{14}$ P. J. Reynolds et al., J. Chem. Phys. 77, 5593 (1982).

${ }^{15}$ B. Králik, A. M. Rappe, and S. G. Louie, Phys. Rev. B 52, 11626 (1995); 56, 4760 (1997). 